\title{
First asteroseismic results from CoRoT
}

\author{
E. Michel ${ }^{1}$, A. Baglin ${ }^{1}$, W.W. Weiss ${ }^{2}$, M. Auvergne ${ }^{1}$, C. Catala ${ }^{1}$, C. Aerts ${ }^{3}$,
} T. Appourchaux ${ }^{4}$, C. Barban ${ }^{1}$, F. Baudin ${ }^{4}$, M. Briquet ${ }^{3}$, F. Carrier ${ }^{3}$, P. Degroote ${ }^{3}$, J. De Ridder ${ }^{3}$, R.A. Garcia ${ }^{5}$, R. Garrido ${ }^{6}$, J. Gutiérrez-Soto ${ }^{1,7}$, T. Kallinger ${ }^{2}$, L. Lefevre ${ }^{1}$, C. Neiner ${ }^{7}$, E. Poretti ${ }^{8}$, R. Samadi ${ }^{1}$, L. Sarro ${ }^{9}$, G. Alecian ${ }^{10}$, L. Andrade ${ }^{11}$, J. Ballot ${ }^{12}$, O. Benomar ${ }^{4}$, G. Berthomieu ${ }^{13}$, P. Boumier ${ }^{4}$, S. Charpinet ${ }^{14}$, B. de Batz ${ }^{7}$, S. Deheuvels ${ }^{1}$, M.-A. Dupret ${ }^{1}$, M. Emilio ${ }^{15}$, J. Fabregat ${ }^{16}$, W. Facanha ${ }^{11}$, M. Floquet ${ }^{7}$, Y. Frémat ${ }^{17}$, M. Fridlund ${ }^{18}$, M.-J. Goupil ${ }^{1}$, A. Grotsch-Noels ${ }^{19}$, G. Handler ${ }^{2}$, A.-L. Huat ${ }^{7}$, A.-M. Hubert ${ }^{7}$, E. Janot-Pacheco ${ }^{11}$, H. Kjeldsen ${ }^{20}$, Y. Lebreton ${ }^{7}$, B. Leroy ${ }^{1}$, C. Martayan ${ }^{7,17}$, P. Mathias ${ }^{21}$, A. Miglio ${ }^{19}$, J. Montalban ${ }^{19}$,

M.J.P.F.G. Monteiro ${ }^{22}$, B. Mosser ${ }^{1}$, J. Provost ${ }^{13}$, C. Regulo ${ }^{23}$, J. Renan de Medeiros $^{24}$, I. Ribas ${ }^{25}$, T. Roca Cortés ${ }^{23}$, I. Roxburgh ${ }^{26,1}$, J. Suso ${ }^{16}$, A. Thoul ${ }^{19}$, T. Toutain ${ }^{27}$, D. Tiphene ${ }^{1}$, S. Turck-Chieze ${ }^{5}$, S. Vauclair ${ }^{14}$, G. Vauclair ${ }^{14}$, K. Zwintz ${ }^{2}$

(Affiliations can be found after the references)

\begin{abstract}
About one year after the end of the first observational run and six months after the first CoRoT data delivery, we comment the data exploitation progress for different types of stars. We consider first results to illustrate how these data of unprecedented quality shed a new light on the field of stellar seismology.

Individual Objects: HD 50747, HD 49933, HD 50890, HD 50170, HD 51106, HD 181420 , HD 181906, HD 180642, HD 181231, HD 175869, HD 181907
\end{abstract}

\section{The present CoRoT harvest}

The detailed description of the instrument and programme has been presented in several places (e.g. Baglin et al. 2006, Auvergne et al. 2006). Detailed measurements of the in-flight performances are now available (Auvergne et al. 2008). We simply remind you here of a few aspects necessary to introduce the results. 
The CoRoT focal plane is divided in two areas of about 4 square degrees on the sky each. One of them (the seismo field) is mostly optimized for the seismology programme (Michel et al. 2006). Ten objects with $5.4<m_{V}<9.5$ can be observed simultaneously with a 1 second-sampling rate and a noise limited by photon noise to $410^{-4}<\sigma<310^{-3}$ per 1 s measurement.

The other field (the exofield) is mostly optimized for the search of exoplanets. About 12,000 objects with $11<m_{V}<15$ can be observed simultaneously, with a $512 \mathrm{~s}$ sampling rate and a standard deviation from $710^{-4}$ to $210^{-3}$ per $512 \mathrm{~s}$ measurement. In this field, for the brightest stars $\left(m_{V}<14.5\right)$, a prism allows to obtain three-colour information. For a limited number $(\sim 500)$, it is possible to select a $32 \mathrm{~s}$ sampling rate.

Altogether, during the 3-years nominal period foreseen for the mission, more than 100 stars will be observed in the Seismo field and more than 100000 in the exofield!

As described in Michel et al. (2006b), the observational programme is composed of long runs (up to 150 days) and short runs (20-30 days). At the time of the Wroctaw conference, 5 runs have been completed successfully: an initial run of 60 days, two long runs and two short runs. The satellite is presently in a long run which will stop by mid-october.

We thus have completed the observations of 50 objects in the seismo field (in fact 49, HD 49933 having been observed twice): 5 solar-like pulsator candidates; 13 B stars, including 1 known Beta Cephei star, 5 Be stars, 2 eclipsing binaries; $7 \mathrm{~F}$ and $\mathrm{G}$ giant stars ; $17 \mathrm{~A}$ stars, including 4 known pulsators, $2 \mathrm{Am}$ stars, 3 Ap stars, 2 eclipsing binaries; 7 early F stars including 2 known Gamma Dor.

A few examples of the light curves are given in Fig. 1 and Fig. 2. They illustrate the quality of the data, revealing in the light curves variability below the $10^{-3}$ level. They also illustrate the variety of variability behaviours encountered.

Solar-like pulsators

The search and characterization of solar-like oscillations in other main sequence stars is one of the highlights and one of the greatest challenges of the CoRoT mission. The main objective is to bring measurements of the modes parameters (frequencies ,amplitudes, mode profiles,...) for objects scanning the Main Sequence in the range of temperature where such oscillations are expected.

An example of light curves obtained for one of these candidates (HD 49933) is given in Fig. 2. In this light curve, the standard deviation of the individual measurements (every $32 \mathrm{~s}$ here) is of the order of $10^{-4}$ (about 100 times below the standard values from ground-based photometry). The light curve already reveals variability of a few $10^{-4}$ which is attributed to activity and interpreted in terms of spots. The solar-like oscillations do not appear in the light curve but are clearly seen in the Fourier spectra (Fig. 3) for three objects of various magnitudes. 

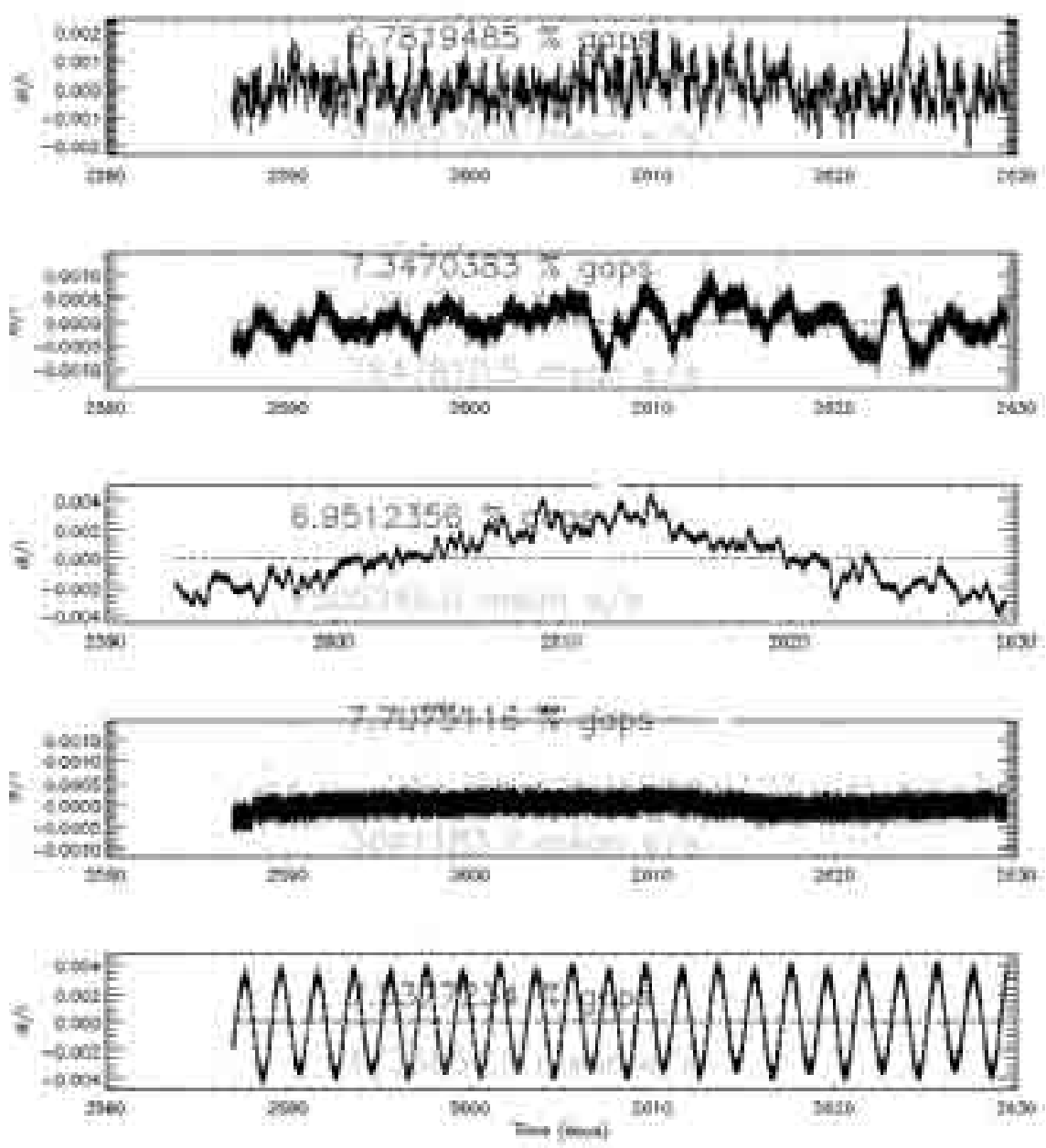

Figure 1: Forty-days-portions of the light curves from 5 stars (top to bottom: the A star HD 50747, the F star HD 49933, the giant G star HD 50890, the giant F star HD 50170, the A star HD 51106)

As shown by Michel et al. (2008) and illustrated in Fig. 3, it is possible to identify three components in the spectra of each of these three stars: (i) a white component compatible with the photon noise; (ii) a component increasing toward low frequencies characterizing the stellar granulation and (iii) the oscillations component with its characteristic comb-like pattern. Michel et al. (2008) give a comparison of these measurements with those observed in the Sun and with theoretical values. 

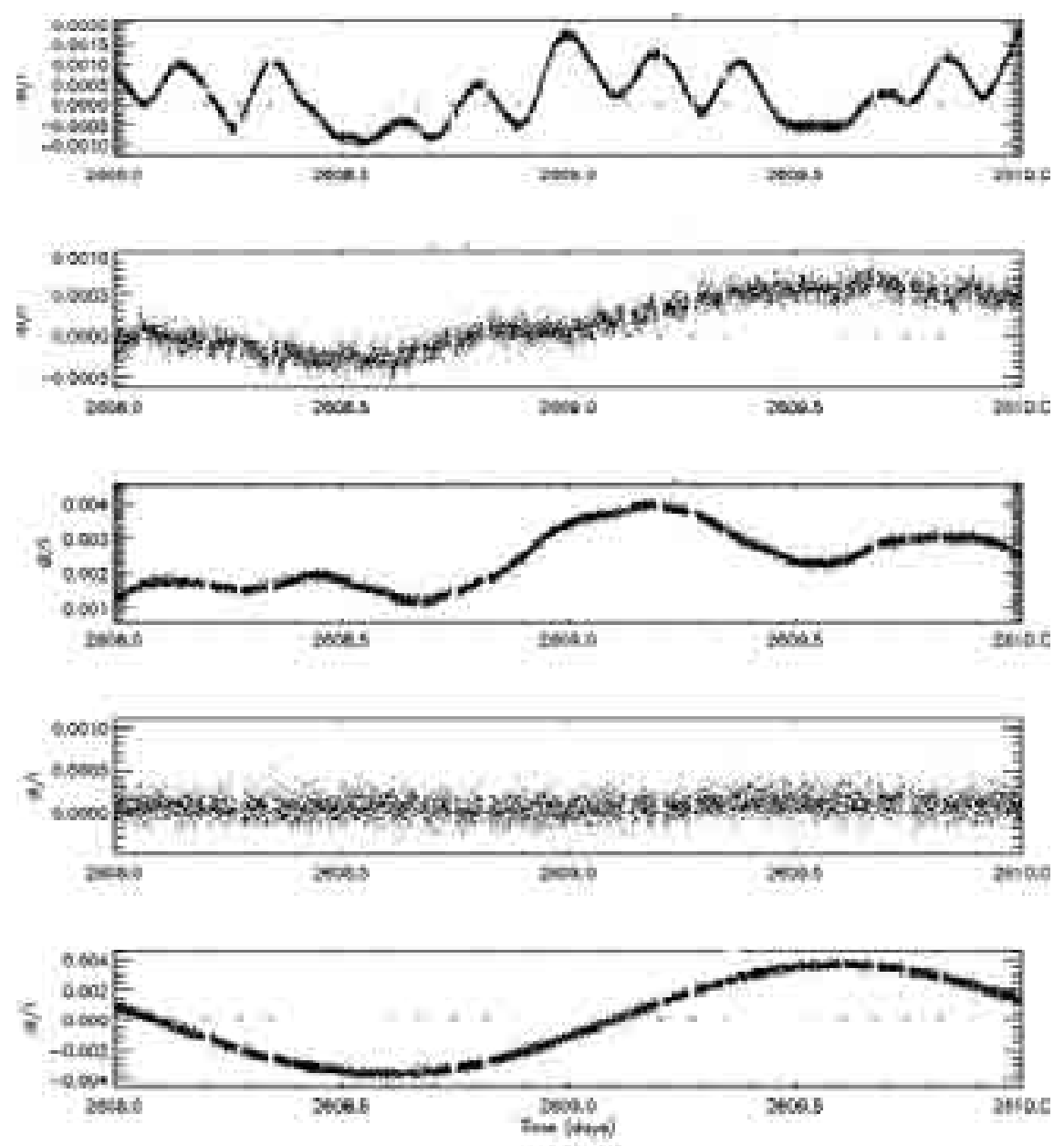

Figure 2: Same as Fig. 1 enlarged over two-days-portions

The activity of data analysis intended to provide for each star individual mode parameters (frequencies, amplitudes, profiles,...) is held within the CoRoT/SWG/DAT (resp. T. Appourchaux, see Appourchaux et al. (2006)). An example of analysis can be found in Appourchaux et al. (2008), where about 40 modes have been characterized for HD 49933, including eigenfrequencies determined with precision of the order of a few $10^{-7} \mathrm{~Hz}$ to a few $10^{-6} \mathrm{~Hz}$ (Fig. 4). Further analyses with alternative methods are under way as illustrated by Garcia et al. (2008). 


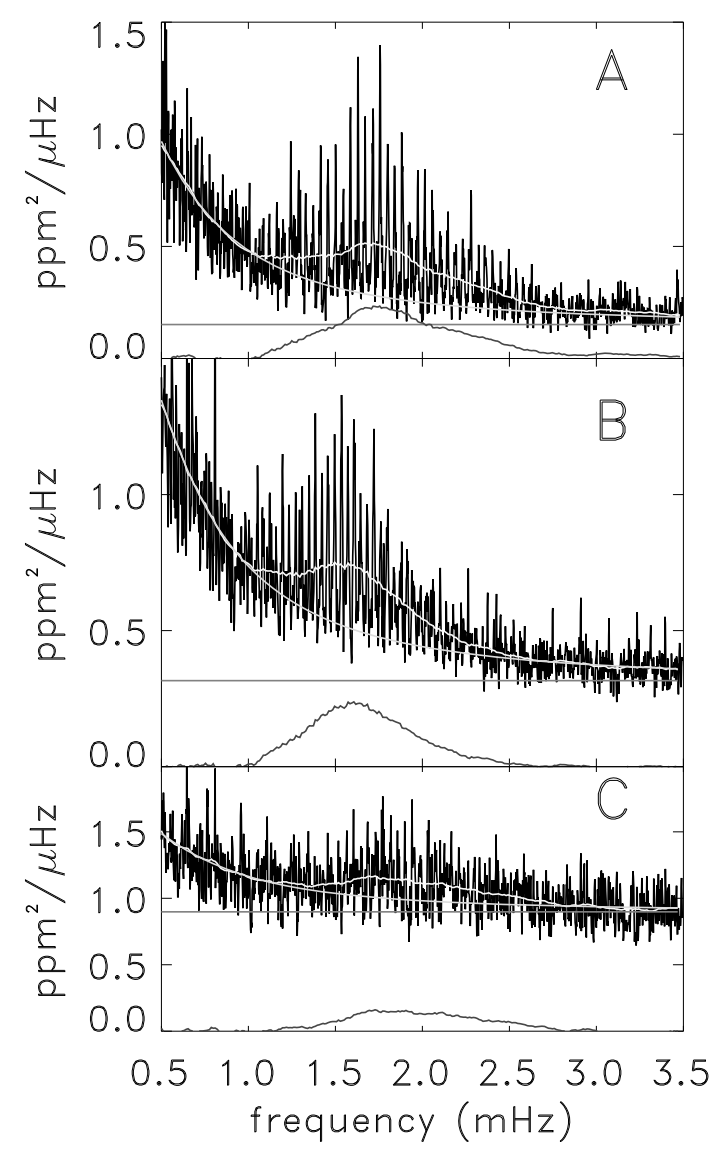

Figure 3: Power spectra of three solar-like pulsators observed with CoRoT: HD 49933 $\left(m_{V}=5.7\right)$, HD $181420\left(m_{V}=6.7\right)$, and HD $181906\left(m_{V}=7.6\right)$. Each spectrum is decomposed in three contributions commented in the text (after Michel et al. 2008)

B stars

As in most of the classical (opacity driven) pulsators, the gain in resolution and noise level is expected to bring a new insight on the pulsational behaviour of the stars and bring precise measurements for seismic studies. In addition, thanks to their continuity, the CoRoT data also allow to tackle oscillations with periods of the order of the day or below, which are very common in Slowly Pulsating B stars and Beta Cephei pulsators. 


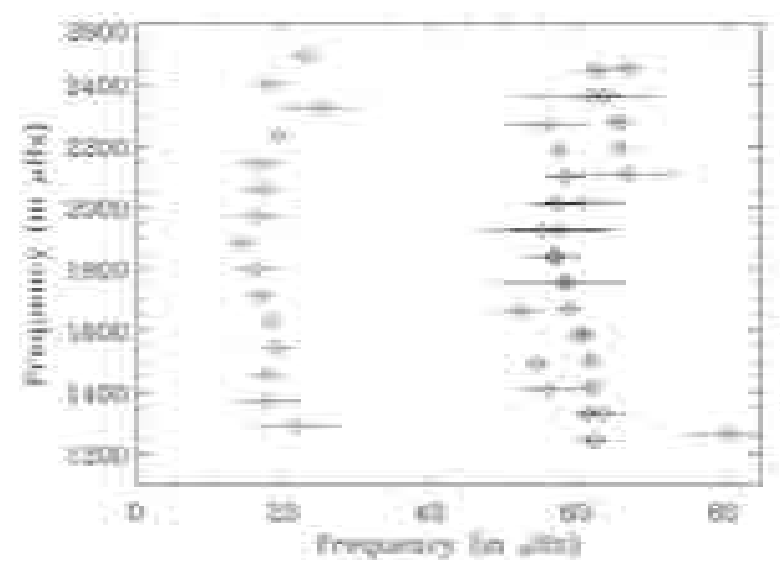

Figure 4: Echelle diagram showing the frequencies of the modes and their 3 sigmaerror bars obtained for HD 49933 (after Appourchaux et al. 2008)

The data analysis of these targets is organized within the CoRoT B stars group (Resp. C. Aerts). As an example, the Fourier spectrum of the known Beta Ceph HD 180642 reveals a dominant radial mode with its harmonics but also low amplitude nonradial modes (Fig. 5).

Among the latter, the highest ones have also been measured in spectroscopy (with FEROS, in the framework of the ESO Large Programme, led by E. Poretti) which will bring helpful complementary information about their identification. More details on this analysis will be given in Briquet et al. (2008).

\section{Be stars}

The analysis lead in the CoRoT/Be stars group (resp. C. Neiner) allowed to reveal oscillations in a B5IVe star (Fig. 6 and Neiner et al. 2008). Only two examples of such oscillation detection in late Be stars existed so far from MOST photometry (Walker et al. 2005, Saio et al. 2007).

For another object, HD 175869, peaks have been detected with amplitudes down to a few $10^{-6}$. A preliminary study suggests that these peaks are associated with oscillations and rotation (Gutierrez-Soto 2008).

$A$ and early $F$ stars

A and early $F$ type stars feature several known classes of variables among which $\delta$ Scuti stars and $\gamma$ Doradus stars are the most famous. For the former, the objects observed from the ground show up to several tens of modes, but always less than expected from theoretical models. This raised the question of the 

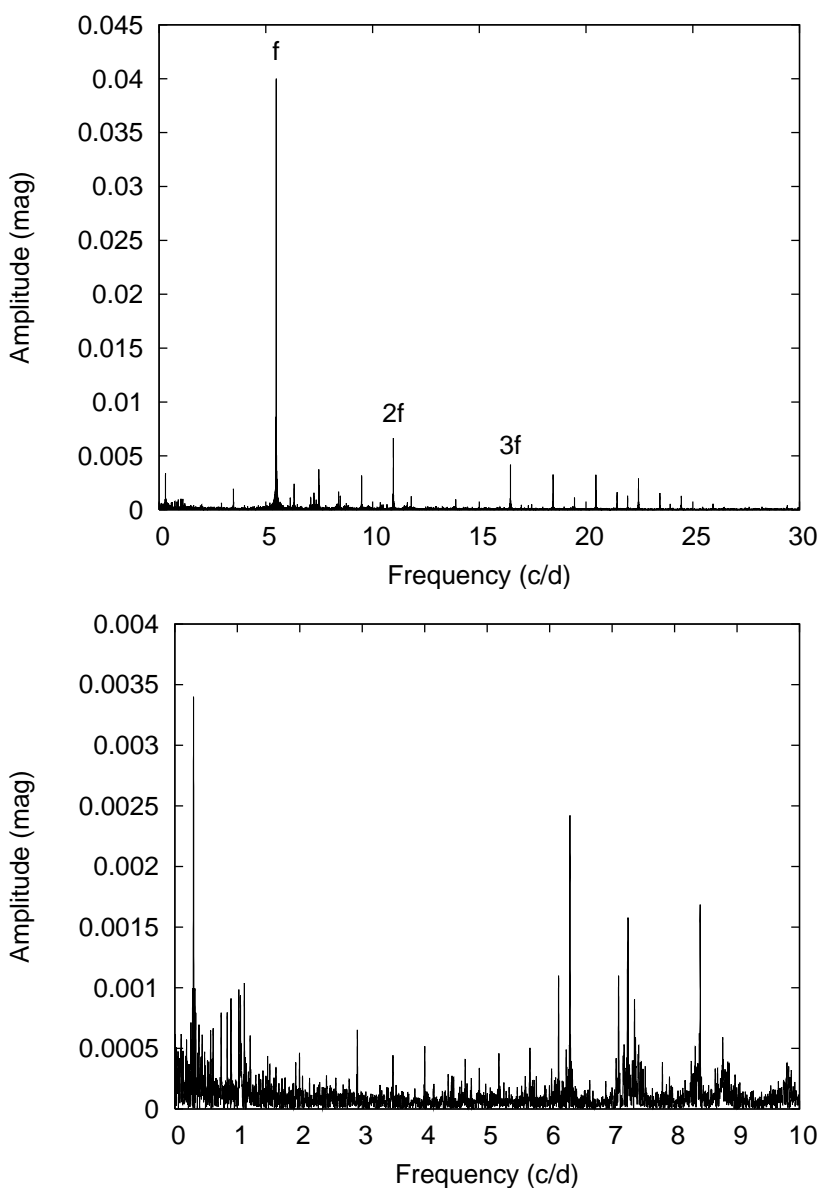

Figure 5: top: Power spectrum of the Beta Cephei HD 180642; bottom: idem after removing the radial mode and its harmonics (after Briquet et al. 2008)

possible existence of selection rules favorising given modes versus others. However, in the existing observations, the detection is always limited in amplitude to a few $10^{-4}$ by the noise level. By lowering the noise level by a factor 100 , the CoRoT data are expected to bring a new insight on this question.

The light curve of HD 174936 reveals variations at the $10^{-3}$ level in the envelope, with beating phenomenon (Fig. 7). The associated Fourier spectrum shown in Fig 8 reveals a very rich spectrum. After fitting and subtracting peaks above an illustrative $410^{-4}$ level, the residual spectrum (Fig 9) reveals 


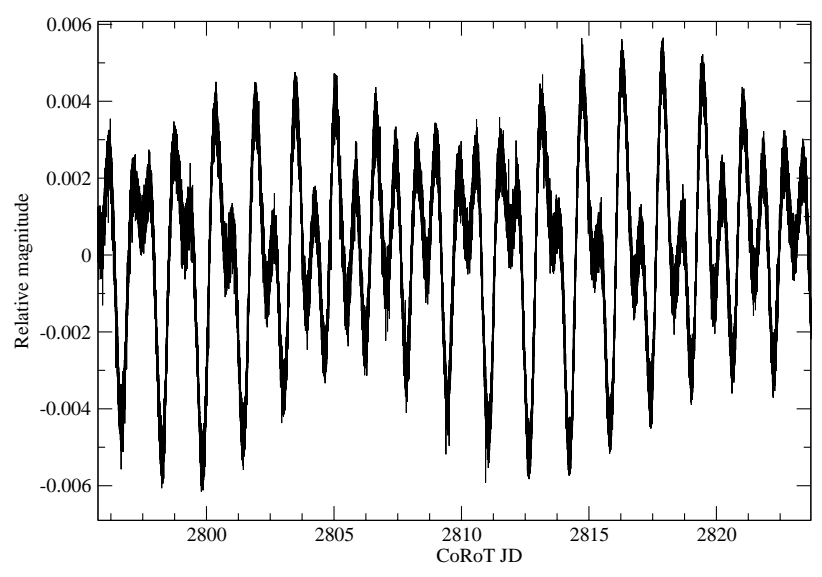

Figure 6: light curve of the B5IVe star HD 181231 (after Neiner et al. 2008)

a high number of remaining peaks, much higher than the noise level as estimated from the Fourier spectrum of a star of same brightness showing no signal in this frequency range. The interpretation of these results is led within the CoRoT/A stars group (resp. R. Garrido and E. Michel).

\section{Giant $\mathrm{F}$ and $\mathrm{G}$ stars}

For these stars, solar-like oscillations are expected with amplitudes significantly larger than in the main sequence solar-like pulsators and with frequencies in the $10-100 \mu \mathrm{Hz}$ domain. Here again, the CoRoT data allow to reveal these oscillations (e.g. Fig. 10).

Some of these objects show more surprising behaviour. One of them (HD 50170) shows no apparent variability in its light curve (Fig. 2). Its spectrum reveals two types of peaks: narrow peaks which would be consistent with long-lived modes forming an $\mathrm{I}=1$ multiplet (Fig. 11) and at a lower amplitude, modes with shorter lieftimes, as suggested by the autocorrelation function (Fig. 11) A paper by Baudin et al., which is in preparation, will discuss this in more detail. This object is being investigated in order to determine whether we are dealing with a new type of hybrid pulsator or these pulsations have to be attributed to separate components of a multiple star. 


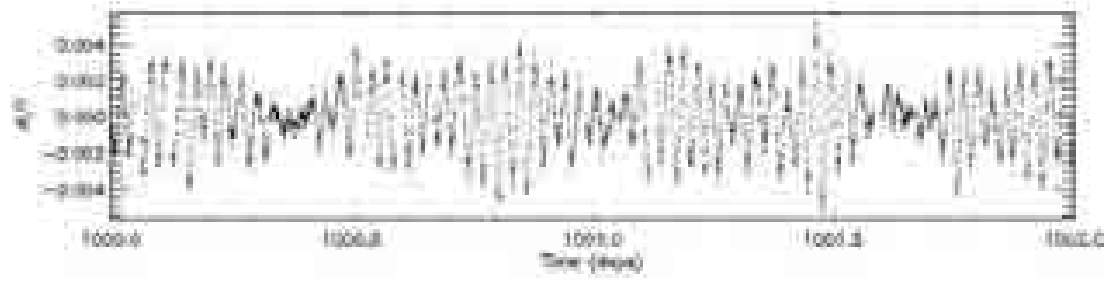

Figure 7: light curve (part of) of the $\delta$ Scuti star HD 174936.

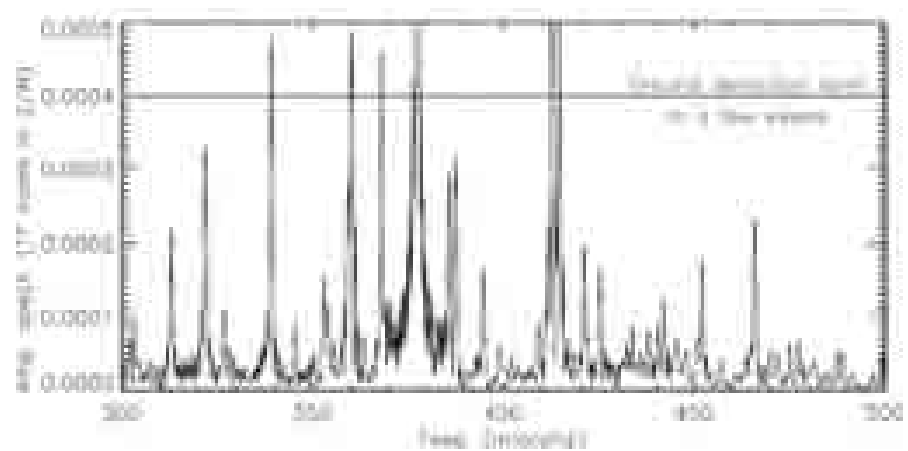

Figure 8: Amplitude spectrum of the $\delta$ Scuti star HD 174936.

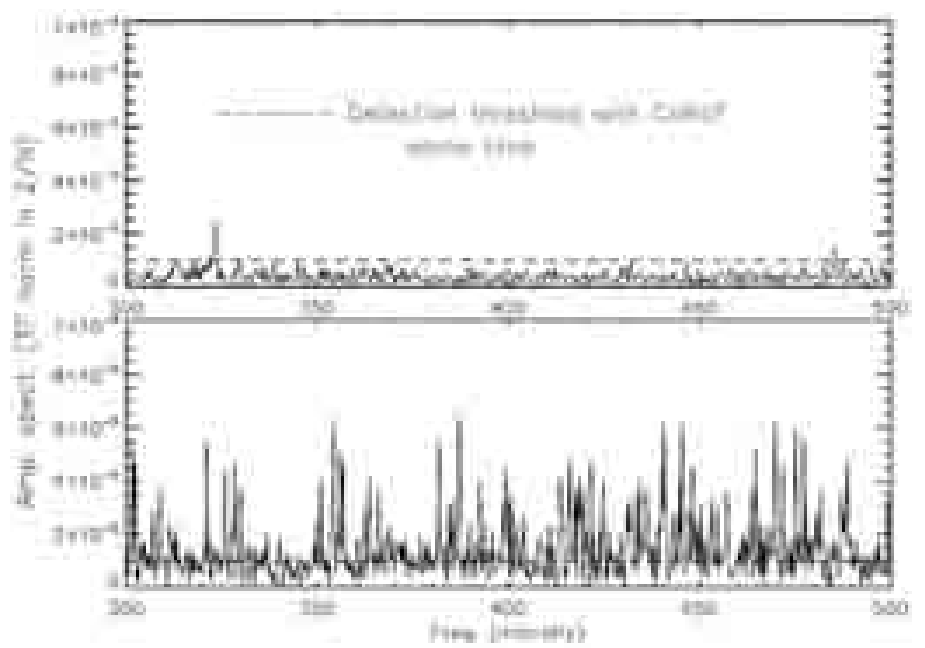

Figure 9: Bottom: same as Fig. 8 after substraction of the highest peaks; top: amplitude spectrum for a comparison star of similar magnitude showing no oscillations in this frequency range 


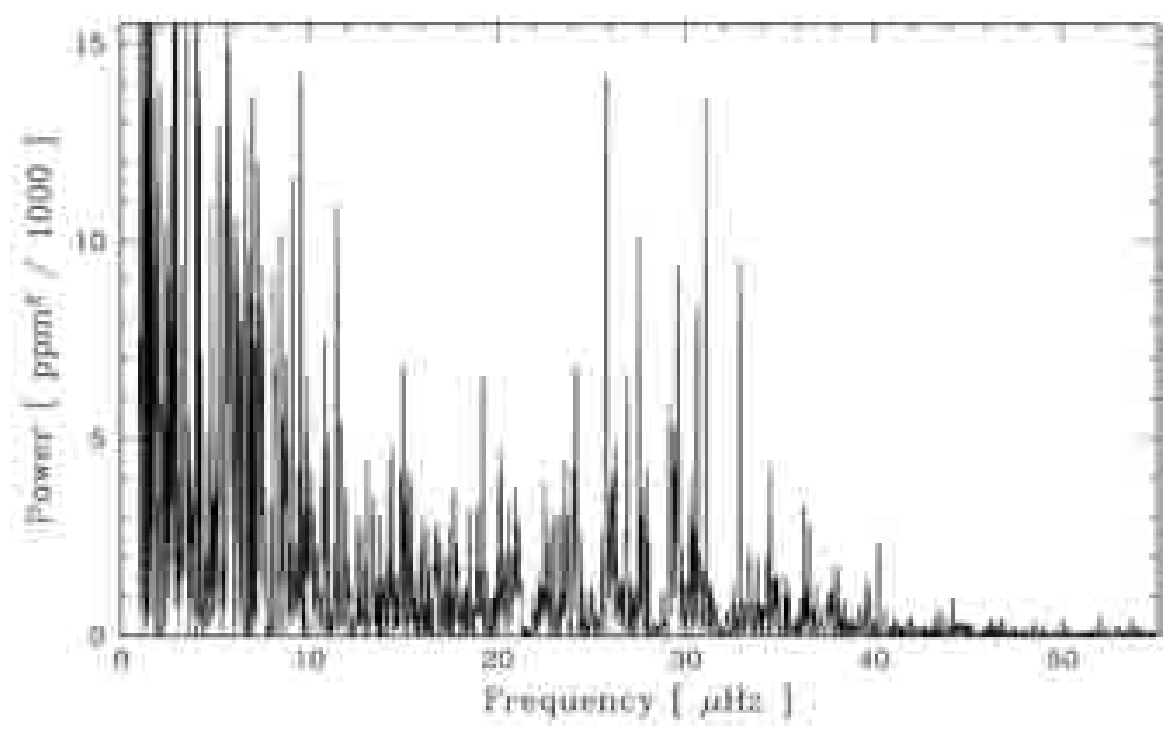

Figure 10: Power spectrum of the giant star HD 181907 showing oscillations in the range $20-40 \mu \mathrm{Hz}$
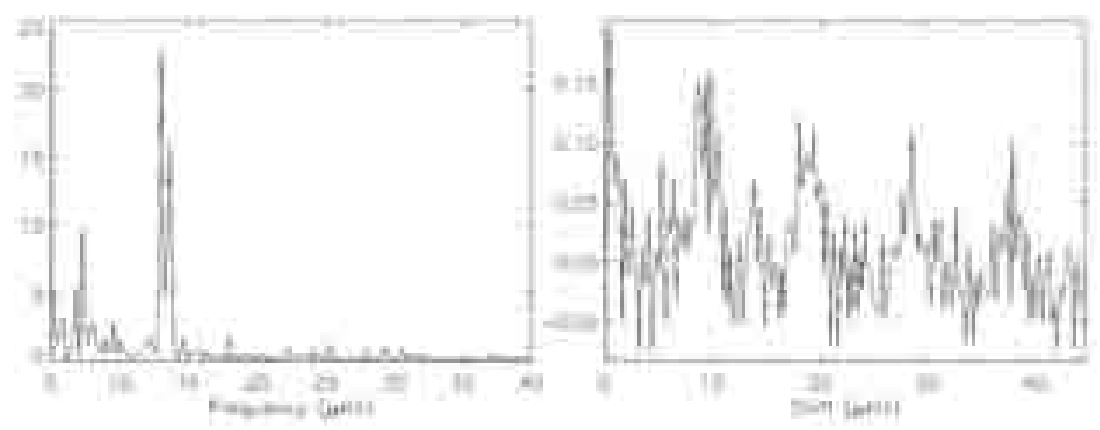

Figure 11: left: Power spectrum of the giant star HD 50170; right: Autocorelation of the power spectrum

In the exofield

About 60000 objects have been observed in the exofield so far. While they are actively searched for occultation due to planet transits, these data also constitute a goldmine for seismology. The activity of seismology on these objects is organized within the Additional Programme Working Group (APWG, led by W. W. Weiss). 

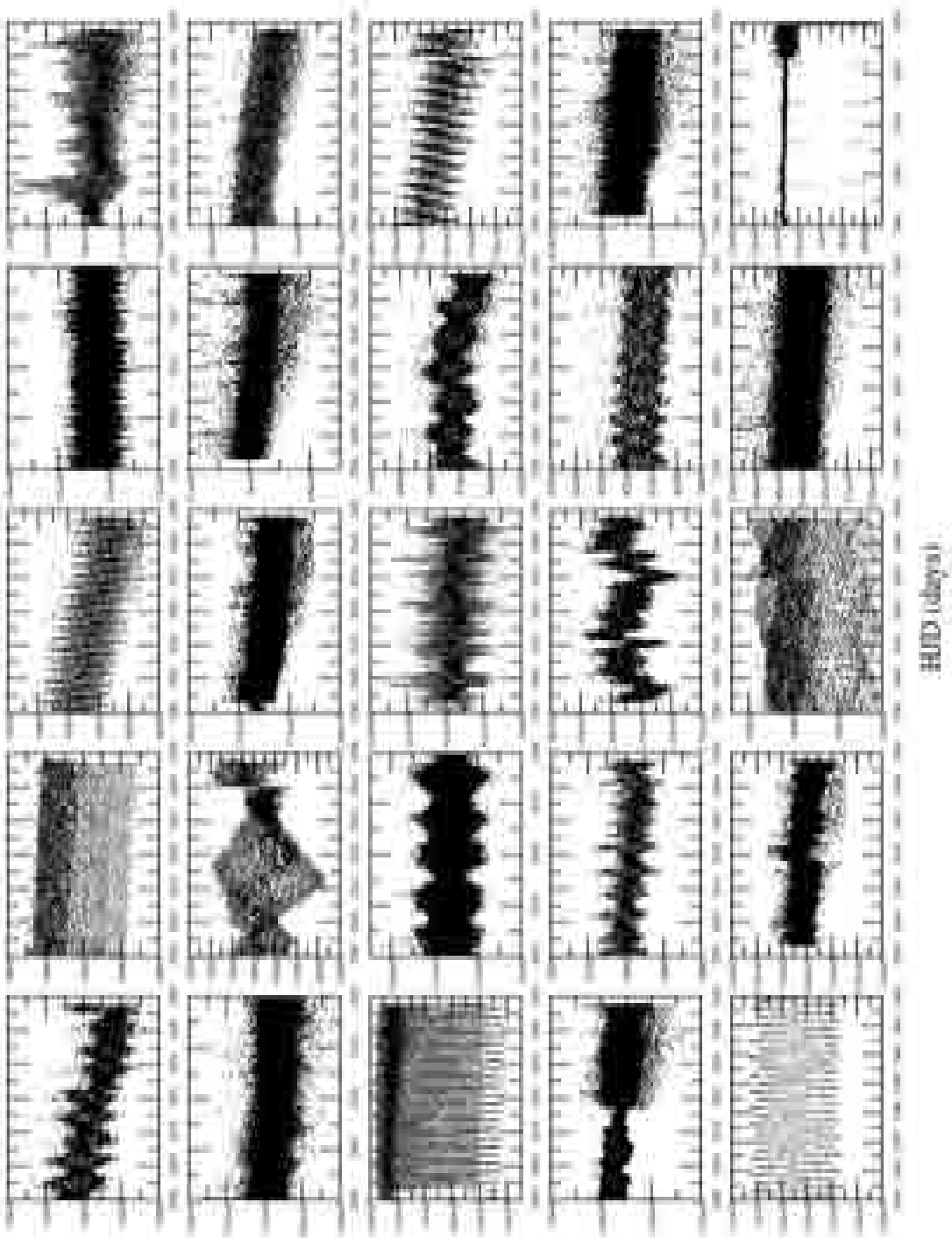

(4)แ⿴囗十) หา1

Figure 12: Sample of light curves obtained in the exofield 
While some of the light curves obtained in the exofield show variations which can easily be recognized as characteristics of specific type of pulsators, others show much more puzzling behaviour (Fig. 12).

Due to the large number of objects considered and to the fact that very few of them have well-defined global parameters (due to their faintness), an activity of automatic classification has been undertaken in two groups (at Leuven and Madrid). These processes are based on self-learning algorithms, and the results obtained so far come from the first loop and are expected to evolve significantly. However, it seems interesting to note that among the few thousands of variables already found, the different known classes of pulsators are represented as well as apparently new classes (Degroote et al. 2008).

The red giants bring a striking example of the potential of the exofield for stellar seismology (led by J. de Ridder). As shown in Kallinger et al. (2008) (see also Hekker et al. 2008), solar-like oscillations are observed in a significant

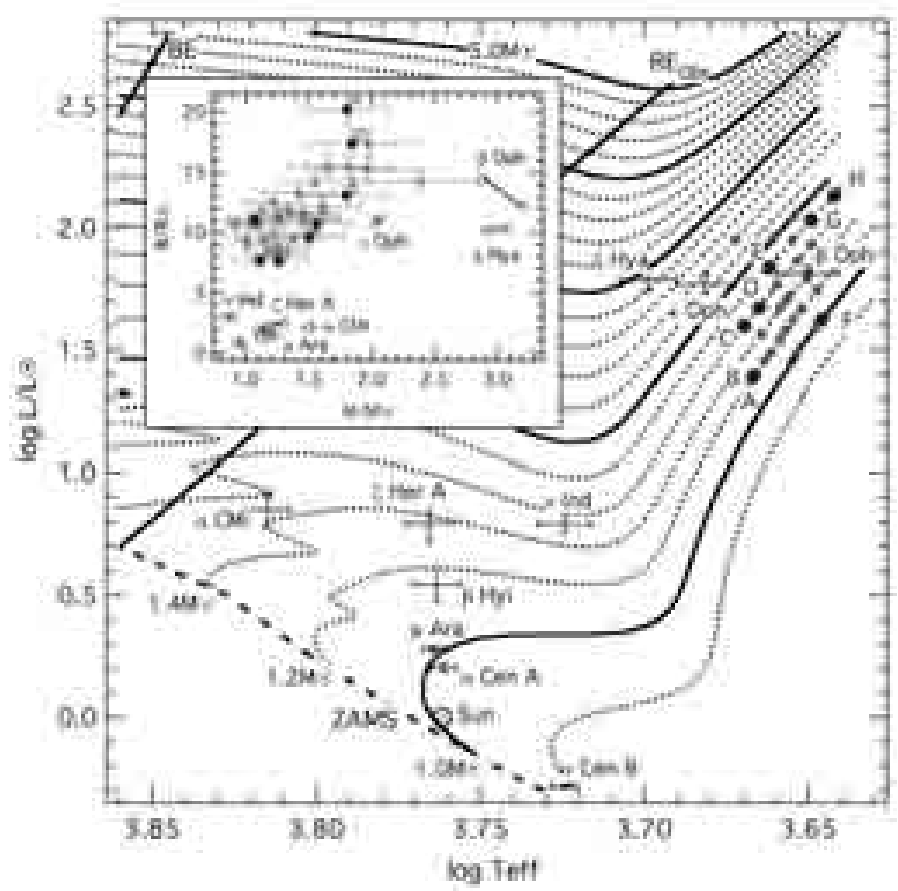

Figure 13: Location of several giant pulsators in an HR diagram and in a mass-radius diagram derived from observed oscillations (after Kallinger et al. 2008) 
number of red giants revealing trends linked with their global parameters. With these data, Kallinger et al. (2008) have been able to estimate mass and radii for those objects (Fig. 13).

\section{Conclusions}

The CoRoT data fulfil expectations in terms of noise level, duration of the runs and continuity of the observations. The analysis of the light curves allows to explore stellar variability at an unprecedented level of precision and for an unprecedented range of time scales. The first interpretation studies confirm the great help we can expect from these data to improve our understanding of stellar structure and evolution. These results also confirm space photometry as an efficient component of the strategy to develop stellar seismology.

Acknowledgments. The CoRoT (Convection Rotation and planetary Transits) space mission, launched on December 2006, was developed and is operated by CNES, with participation of the Science Program of ESA, ESA's RSSD, Austria, Belgium, Brazil, Germany and Spain. The Austrian Science Fonds has supported WWW and TK (P17890), and KZ (T335-N16). MB is Postdoctoral Fellow of the Fund for Scientific Research, Flandres.

\section{References}

Appourchaux, T., Berthomieu, G., Michel, E., et al. 2006, The CoRoT Mission, Eds.

Fridlund M., Baglin A., Lochard J. and L. Conroy, ESA SP-1306, 377

Appourchaux, T., Michel, E., Auvergne, M., et al. 2008, A\&A, 488, 705

Auvergne, M. 2006, The CoRoT Mission, Eds. Fridlund M., Baglin A., Lochard J. and L. Conroy, ESA SP-1306, 283

Auvergne, M., Boisnard, L., Lam-Trong, T., et al. 2008, A\&A, in press

Baglin, A., Auvergne, M., Barge, P., et al. 2006, The CoRoT Mission, Eds. Fridlund M., Baglin A., Lochard J. and L. Conroy, ESA SP-1306, 33

Briquet, M., Uytterhoeven, K., Aerts, C., et al. 2008, CoAst, 158, in press

Degroote, P., Miglio, A., Debosscher, J., et al. 2008, CoAst, 158, in press

Garcia, R., Appourchaux, T., Baglin, A., et al. 2008, CoAst, 157, in press

Gutierrez-Soto, J., Neiner, C., Hubert, A-M., et al. 2008, CoAst, 157, in press

Hekker, S., Barban, C., Hattzes, A., et al. 2008, CoAst, 157, in press

Kallinger, T., Weiss, W. W., Barban, C., et al. 2008, A\&A, in press

Michel, E., Baglin, A., Auvergne, M., et al. 2006, The CoRoT Mission, Eds. Fridlund M., Baglin A., Lochard J. and L. Conroy, ESA SP-1306, 39

Michel, E., Deleuil, M., \& Baglin, A. 2006b, The CoRoT Mission, Eds. Fridlund M., Baglin A., Lochard J. and L. Conroy, ESA SP-1306, 473 
Michel, E., Baglin, A., Auvergne, M., et al. 2008, Science, 322, 558

Neiner, C., Gutierrez-Soto, J, Fremat, Y., et al. 2008, CoAst, 157, in press

Saio, H., Cameron, C., Kuschnig, R., et al. 2007, ApJ, 654, 544

Walker, G. A. H., Kuschnig, R., Matthews, J. M., et al. 2005, A\&A, 635, 77

\section{Affiliations of the authors}

1 LESIA-Observatoire de Paris-CNRS (UMR 8109)-Univ. Paris 6- Univ. Paris 7, pl. J. Janssen, F-92195 Meudon, France

2 Institute for Astronomy Univ. of Vienna, Tuerkenschanzstrasse 17, A-1180 Vienna, Austria

3 Instituut voor Sterrenkunde, Katholieke Univ. Leuven, Celestijnenlaan 200 D, B-3001 Leuven, Belgium

4 Institut d'Astrophysique Spatial, Univ. Paris 11 - CNRS (UMR 8617), F-91405 Orsay, France

5 Labo. AIM, CEA/DSM-CNRS-Univ. Paris 7; CEA, IRFU, SAp, Centre Saclay F-91191 Gif-sur-Yvette, France

6 Instituto de Astrofisica de Andalousia -CSIC, Camino Bajo de Huetor, 50, E-18008 Granada, Spain

7 GEPI-Observatoire de Paris-CNRS (UMR 8111)- Univ. Paris 7 , pl. J. Janssen, F-92195 Meudon, France

8 INAF-Osservatorio Astron. di Brera, Via E. Bianchi 46, I-23807 Merate (LC) Italy

9 LAEFF, Apt. 78, E-28691 Villanueva de la Cañada Madrid, Spain

10 LUTH-Observatoire de Paris-CNRS-Univ. Paris 7, pl. J. Janssen, F-92195 Meudon, France

11 Universidade de Sao Paulo, Rua do Matão, 1226 São Paulo-SP Brazil

12 Max Planck Inst. für Astrophysik, Karl-Schwarzschild-Str. 1, Postfach 1317, 85741 Garching, Germany

13 Laboratoire Cassiopée, Observatoire de la Côte d'Azur-Univ. Nice-Sophia Antipolis-CNRS (UMR 6202), BP 4229, F-06304 Nice, France

14 Laboratoire d'Astrophysique de Toulouse-Tarbes- Univ. de Toulouse-CNRS, 14, av. E. Belin, F-31400 Toulouse, France

15 Universidade Estadual de Ponta Grossa, Praca Santos Andrade, 1, Ponta Grossa- Paraná- Brazil

16 Observatori Astronómic de la Universitat de Valéncia, Poligon La Coma, 46980 Paterna Valéncia 
17 Royal Observatory of Belgium, 3 av. circulaire, 1180 Brussel, Belgium

18 Astrophysics Mission Division, RSSD ESA, ASTEC, SCI-SA P.O. Box 2999, Keplerlaan 1 NL-2200AG, Noordwijk, The Netherlands

19 Department of Astrophysics and Geophysics-FNRS, Liège Univ., 17 allée du 6 août, 4000 Liège, Belgium

20 Danish AsteroSeismology Centre (DASC), Department of Physics and Astronomy, Univ. of AArhus, 8000 Aarhus C, Denmark

21 Laboratoire Fizeau, Observatoire de la Côte d'Azur-CNRS (UMR 6525)Univ. Nice Sophia Antipolis, campus Valrose, F-06108 Nice, France

22 DMA/FCUP \& Centro de Astrofísica da Univ. do Porto, Ruas das Estrelas, 4150-762, Porto, Portugal

23 Instituto de Astrofísica de Canarias - Departamento de Astrofísica, Univ. de La Laguna, Tenerife, Spain

24 Departamento de Física, Univ. Federal do Rio Grande do Norte, 59072-970, Natal RN, Brasil

25 Institut de Ciencies de l'Espai (CSIC-IEEC) Campus UAB Facultat de Ciències, Torre C5-parell, 2a pl 08193 Bellaterra, Spain

26 Astronomy Unit, Queen Mary, Univ. of London, Mile End Road, London E1 4NS, UK

27 School of Physics and Astronomy, Univ. of Birmingham, Edgbaston B15 2TT, UK 
